\title{
JOURNAL OF AUTOMATION AND INFORMATION SCIENCES
}

\section{CONTENTS}

1 Determination of Optimal Routes of Single-Frame Shooting by Spacecrafts and Their Clusters

V.M. Kuntsevich

9 Fast Simulation of Reliability of Repairable System Operating in Two Modes N.Yu. Kuznetsov \& O.N. Homyak

24 On Discrete Conflict-Controlled Processes Described by Grunvald-Letnikov Fractional Systems

V.J. Zhukovskiy \& A.A. Chikriy

35 Stability of the Crystallization Front in a Prizmatical Ampule under Fast Vibration Yu.P. Ladikov-Roev \& O.K. Cheremnykh

51 Zonal Control Synthesis for Nonlinear Systems under Nonlinear Output Feedback K.R. Aida-zade \& S.Z. Guliyev

67 Laplace-Stieltjes Transform for Distribution of Semi-Markovian Chance Path Process with Reflecting Barrier at Zero

T.I. Nasirova, E.M. Neymanov, \& E.A. Ibayev

77 Profit Optimization in Virtual Business Applying Fuzzy Logic

E.R. Shafizadeh \& R.Yu. Shikhlinskaya 


\section{CONTENTS}

1 Modeling of Wave Regimes and Control Parameters at Body Motion under the Water I.T. Selezov, Iu.G. Kryvonos, \& O.V. Avramenko

14 Stabilization of Nonlinear Systems State Observers under Uncertainty S.M. Onishchenko

25 On Determining the Parameters of Sinusoids A.S. Apostolyuk \& V.B. Larin

35 Linear-Quadratic Optimal Control Problem for a Hyperbolic System M.M. Kopets

49 Solving the Safe Problem on Matrixes with Two Types of Locks Aghaei Agh Ghamish Yaghaub

56 Problem of Distribution and Routing of Transport Blocks with Mixed Attachments and Its Decomposition

V.A. Vasyanin

70 On the Problem of Stabilization of Stochastic Differential-Functional Equations with Impulse Markovian Perturbations and Constant Lag. Part III

V.I. Musurivskiy \& V.K. Yasinskiy

77 Discretization of Continuum Antagonistic Game on Unit Hypercube and Transformation of Multidimensional Matrix for Solving of the Corresponding Matrix Game

V.V. Romanuke 


\section{CONTENTS}

1 Guaranteed State Estimation Algorithms for Linear Systems in the Presence of Bounded Noise

V.F. Gubarev \& S.V. Melnychuk

11 Adaptive Control Method for Unstable Impulse Processes in Cognitive Maps Based on Reference Model

V.D. Romanenko, Yu.L. Milyavsky, \& A.A. Reutov

24 Generalized Method of Processing Cyclic Signals of Complex Form in Multidimensional Space of Parameters

L.S. Fainzilberg

40 Project of Eurasian Segment of the New System of Forest Fire Risk Prediction Based on Information and Computer Technologies

N. V. Baranovskiy

57 Linear Complexity Profile as a Means of Measuring the Quality of Random Sequences L.A. Zavadska \& M.A. Semybalamut

71 Analytical reconstruction of the $\boldsymbol{R}$-class systems

V.G. Gorodetskyi \& N.P. Osadchuk 


\section{CONTENTS}

1 On Mechanism of Recession Phenomenon

N.S. Gonchar, A.S. Zhokhin, \& V.G. Kozyrski

18 Modeling the Dynamics of Ecological-Economic Systems Based on Economic Structuring a Society. Part I

V.S. Grygorkiv \& M.V. Grygorkiv

28 Prediction of "Structure-Property" Dependence of New Organic Compounds on the Basis of Artificial Immune Systems

G.A. Samigulina, Z.I. Samigulina, W. Wojcik, \& Yu.V. Krak

36 Application of the Extended $\chi^{2}$-Criterion for Embedded Information Detecting L.L. Nikitenko

43 Differential Games of Fractional Order with Impulse Effect

I.I. Matychyn \& V.V. Onyshchenko

54 Hierarchical Information-Extreme Learning Algorithm for a Diagnostic System with Redundant Training Matrix

A.S. Dovbysh, A.A. Stadnyk, \& S.A. Holubnychyi

64 The Mathematical Modeling of the Human Gait with Ankle Hinged Orthosis M.V. Demydyuk \& B.A. Lytwyn

78 Separation of Convex Hulls as a Way for Modeling of Systems of Prediction of Complications in Patients

M.A. Ivanchuk \& I.V. Malyk 


\section{CONTENTS}

1 Optimal Stabilization of a Simple Pendulum in the Unstable Equilibrium Position S.M. Onishchenko

19 Calculation of the Characteristics of the Multichannel Queueing System with Pure Losses and Feedback

A.Z. Melikov, L.A. Ponomarenko, \& Kh.N. Kuliyeva

30 Algorithms of the Attitude Matching of Star Tracker and Camera of Spacecraft

A.I. Tkachenko

42 State Estimation of Distributed Parameters Systems Using Finite-Dimensional Models and Local Measurements with Bounded Noise

N.N. Salnikov \& A.N. Salnikova

60 The Inverse Steganalysis Methods Based on $\chi^{2}$-Criterion

L.L. Nikitenko

69 On Solving of a Special Optimization Problem Connected with Determination of Invariant Sets of Dynamical Systems

O.A. Berezovskyi

78 The Complexity of Probabilistic Stability Analysis Procedures for Integral Boolean Programming Problems

N.V. Lishchuk

85 Chronicle 


\section{CONTENTS}

1 Synthesis of Control Algorithms of the Spacecraft Spatial Reorientation with the Use of Dynamic Equations of a Solid Body Rotational Motion in Rodrigo-Hamilton Parameters N.V. Yefimenko

17 The Method of Control of Nuclear Power Plant with VVER-1000 Reactor in Maneuverable Mode

M.V. Maksimov, T.A. Tsiselskaya, \& E.A. Kokol

33 Modeling the Dynamics of Ecological-Economic Systems Based on Economic Structuring a Society. Part II

V.S. Grygorkiv \& M.V. Grygorkiv

41 Stochastic Model of an Optimal Single-Component Economics of Growth in Nonlinear Ecological-Economic Criterion with Wiener and Poisson Processes

M.V. Boychuk \& A.R. Semchuk

57 Necessary Conditions of Optimality of the First and Second Order in a Stepwise Optimal Control Problem with Discrete-Continuous System

R.O. Mastaliyev

70 Optimal Control of Process of Oscillations of Thin Rectangular Beam M.M. Kopets 


\section{CONTENTS}

1 Our Hero of the Anniversary

4 On the Modeling of Fractional-Differential Dynamics of Some Processes of Relaxational Filtration

V.M. Bulavatsky \& Iu.G. Kryvonos

15 Simulation of Packing, Distribution and Routing of Small-Size Discrete Flows in a Multicommodity Network

A.N. Trofimchuk \& V.A. Vasyanin

31 A Strongly Convergent Modified Extragradient Method for Variational Inequalities with Non-Lipschitz Operators

D.A. Verlan, V.V. Semenov, \& L.M. Chabak

47 Assessment of Accuracy in the Process of Adjustment of the Identification Matrix V.A. Voronov, A.V. Lakeyev, Yu.E. Linke, \& V.A. Rusanov

59 Modeling in a Class of Autoregression Equations Systems in Conditions of Structural Uncertainty

A.P. Sarychev 


\section{CONTENTS}

1 The Research of the Asymptotic Properties of Nonparametric Classifiers Based on Depth Functions

A.V. Anisimov \& A.A. Galkin

11 Geoinformation Monitoring of Forest Fire Danger on the Basis of Remote Sensing Data of Surface by the Artificial Earth Satellite

N.V. Baranovskiy \& E.P. Yankovich

24 Analytical Representation of the Density Function of Normal Distribution of Noise T.A. Aliev, N.F. Musaeva, M.T. Suleymanova, \& B.I. Gazizade

41 Model and Numerical Solving Algorithm of Counteraction Problem for Two Restored after Failure Redundant Engineering Systems

V.I. Potapov

52 Using Genetic Algorithm for Solving Boundary Value Problems L.P. Vakal

63 Modified Frequency Method of Structural-Parametric Identification of Systems S.V. Melnychuk

74 Mean Time of Approach in Game Problems with Random Excitations V.K. Chikrii 


\section{CONTENTS}

1 On Solution of the Linear Matrix Equations

V.B. Larin

10 Acoustic-Gravity Waves in Whirling Polar Thermosphere

Yu.P. Ladikov-Royev, O.K. Cheremnykh, A.K. Fedorenko, \& V.E. Nabivach

23 Analysis of Queuing System with Dynamic Priorities

A.Z. Melikov, L.A. Ponomarenko, \& B.G. Ismailov

34 The Discrete Frequency Responses of Continuous Linear Systems

N.A. Balonin, V.S. Suzdal, \& A.V. Sobolev

42 Implementation of the Finite Element Method with Optimal Choice of Basic Functions for Dirichlet Problem for Poisson Equation

O.N. Lytvyn, K.V. Nosov, \& T.A. Baranova

63 Numerical Method of Integrating the Variational Equations for Cauchy Problem Based on Differential Transformations

M.Yu. Rakushev

76 Uniform Sampling of Fundamental Simplexes as Sets of Players' Mixed Strategies in the Finite Noncooperative Game for Finding Equilibrium Situations with Possible Concessions

V.V. Romanuke 


\section{CONTENTS}

1 Model of Autocorrelative Function of Time Series with Strong Dependence N.D. Pankratova \& N.G. Zrazhevska

13 Data Analysis Method and Problems of Identification of Trajectories of Solitary Waves A.Ya. Bomba \& Yu.V. Turbal

24 Localization and Recognition of Vehicle Number Plates by Means of the Method of Support Vector Machine and Histograms of Oriented Gradients

A.S. Tlebaldinova, Yu.V. Krak, A.V. Barmak, \& N.F. Denisova

32 Correction of Pedestrian SDINS in a Stay Mode

A.I. Tkachenko

45 Matching of External Criterion and Method of Sample Partitioning for Solving Problem of Structural-Parametric Identification by Group Method of Data Handling

N.V. Kondrashova

60 Application of Smoothing Measures in Nonparametric Kernel Classifiers with Usage of Normal Approximation of Probabilities

A.A. Galkin

69 Method for Evaluation of the Uncertainty of the Paired Comparisons Expert Judgements when Calculating the Decision Alternatives Weights

N.I. Nedashkovskaya

83 Information Material 


\section{CONTENTS}

$1 \quad$ Parametric Synthesis of Digital Stabilization System of Tank Gun

Ye. Ye. Aleksandrov \& T. Ye. Aleksandrova

18 Investigation of Continuous Systems Oscillatory Processes Created with the Multiplicity Factor of the Eigenvalues of the State Matrices

N.A. Vunder \& A.V. Ushakov

36 Suboptimal Nonlinear Control for Operation Speed Criterion on the Basis of Invariant Immersion Method

V.F. Kudin \& B.I. Pryymak

48 Strong Global Attractor for the Three-Dimensional Navier-Stokes System of Equations in Unbounded Domain of Channel Type

N.V. Gorban, A.V. Kapustyan, E.A. Kapustyan, \& O.V. Khomenko

60 Development of the Methodology of Estimating of the Effectiveness in the Space Activity L.I. Samoilenko \& L.N. Kolos

74 On Nonstationary Game Problem of Motion Control

Al.A. Chikrii 


\section{CONTENTS}

1 On a Problem of Numerical Simulating the Derivative of Discrete Time Series with Approximate Values

E.M. Kiseleva, L.L. Hart, \& P.A. Dovgay

18 Predictive Control of Nonlinear Objects Using Evolving Feedforward Neural Networks O.G. Rudenko \& A.A. Bezsonov

29 Methods and Tools for Text Analysis of Publications to Study the Functioning of Scientific Schools

N.B. Shakhovska \& R.Yu. Noha

44 On the Properties and Estimates for Prime Numbers Sequences

E. V. Ivokhin \& D.A. Vadnev

59 On Some Results of Mathematical Modeling of Solutions of Control Problems of Dynamics of Spatially Distributed Processes

V.A. Stoyan

72 Contents 2015 